\title{
A Rare Case of Cor Triatriatum Sinister in Adulthood with Atypical Manifestation
}

\author{
Thauler Alves de Oliveira ${ }^{1}$ and Renan Attílio Santos Marquiori ${ }^{2}$ \\ 1. School of Medicine, University of Itaúna, Itaúna, Minas Gerais, Brazil; 2. Department of Cardiology, Santa Casa de Misericórdia Hospital, \\ Belo Horizonte, Minas Gerais, Brazil
}

\begin{abstract}
A 47-year-old man was admitted to the Santa Casa de Misericórdia Hospital in Belo Horizonte, Brazil, with recurrent signs and symptoms of tachycardia, palpitation and fatigue. During medical examination, an electrocardiogram and 24-hour Holter monitoring were performed, which identified a predominant atrial flutter rhythm and, after transthoracic echocardiography, the patient was diagnosed with cor triatriatum sinister. The condition is a rare congenital heart disease characterised by the presence of a fibrous membrane that divides the left atrium into two separate chambers. The disease is especially evident during childhood; however, some cases show no signs until adulthood, which makes the presentation even more unique. In this case, clinical medication and electrical cardioversion were chosen as treatments, which caused progression to sinus rhythm and improvement of symptoms. Therefore, due to the stability of the condition, continuous follow-up with a cardiologist was implemented.
\end{abstract}

\section{Keywords}

Cor triatriatum, cor triatriatum sinister, congenital heart disease, heart anomalies

Disclosures: Thauler Alves de Oliveira and Renan Attílio Santos Marquiori have no financial or non-financial relationships or activities to declare in relation to this article.

Review Process: Double-blind peer review.

Compliance with Ethics: Informed consent was received from the patient in this case study. Research and Ethics Committee, number 5138 Santa Casa de Misericórdia of Belo Horizonte, Minas Gerais, Brazil. Brazilian National Register of Legal Entities: 17.209.891/0001-93. Technical evaluation: approved; technical evaluation number: 3.321.316, CAAE: 07220819.0.0000.5138.

Authorship: The named authors meet the Internationa Committee of Medical Journal Editors (ICMJE) criteria for authorship of this manuscript, take responsibility for the integrity of the work as a whole, and have given final approval for the version to be published.

Access: This article is freely accessible at touchCARDIO.com (c) Touch Medical Media 2020.

Received: 27 January 2020

Accepted: 27 April 2020

Published Online: 14 June 2020

Citation: Heart International. 2020;14(1):53-5

Corresponding Author: Thauler Alves de Oliveira, School of Medicine, University of Itaúna, João Dornas,

47, Itaúna, Minas Gerais, 35680335, Brazil.

E: thauleralves@gmail.com

Support: No funding was received in

the publication of this article.
First described in 1868 by WS Church, cor triatriatum sinister (CTS) is one of the rarest congenital heart diseases in the world, with an estimated incidence of $0.1 \%{ }^{1.2}$ It is characterised by the presence of a fibrous membrane that divides the left atrium into two different regions: a proximal chamber with the pulmonary veins and another distal chamber with the mitral valve and the left atrial appendage. ${ }^{3}$ There are several theories regarding its cause, with anomalous growth of the septum primum during embryogenesis being the most widely cited. ${ }^{4-8}$ This disease is especially evident during childhood; however, some cases show no signs until adulthood. The most common symptoms are dyspnoea, haemoptysis, orthopnoea and chest pain. ${ }^{\circ}$ Symptom severity in CTS is variable, which also affects the recommended treatment options. For patients with late diagnosis and less severe obstruction, surgery may be unnecessary. ${ }^{10}$ Currently, there are no formal guidelines concerning the ideal timeframe for surgical correction, owing to the rarity of the disease.11

\section{Case report}

A previously healthy 47-year-old male sought outpatient public healthcare for dyspnoea associated with fatigue and palpitation. Electrocardiogram (ECG) showed atrial flutter and the patient was transferred to intermediate hospital healthcare for heart-rate control. After beta-blocker and anticoagulant therapy, the patient developed clinical improvement and was discharged from hospital with outpatient follow-up. Thirty days later, at a follow-up appointment, 24-hour Holter monitoring was performed, which showed atrial flutter with a variable degree of atrioventricular block. As the symptoms persisted, the patient sought emergency medical care again and the patient was transferred to advanced hospital care. Physical examination was performed, which did not reveal any abnormalities. ECG (Figure 1) showed atypical atrial flutter with variable atrioventricular block, and chest X-ray (Figure 2) revealed normal structures. Transthoracic echocardiogram with Doppler (Figures 3 and 4) showed normal dimensions in the left atrium and the presence of a filamentous membrane subdividing the atrium, with a flow passage through the central portion - apparently single foramen - without generating significant gradient (peak gradient $7.63 \mathrm{mmHg}$ and mean $3.2 \mathrm{mmHg}$, mean velocity $1.2 \mathrm{~m} / \mathrm{s}$ ). This was suggestive of CTS. The echocardiogram also showed the left ventricle had no segmental deficit; however, there was mild mitral regurgitation and tricuspid valve with prolapse and mild tricuspid regurgitation. Pulmonary artery systolic pressure $25 \mathrm{mmHg}$. Transoesophageal echocardiography revealed the absence of thrombi in the left atrium and the presence of a left intra-atrial membrane, suggestive of non-restrictive CTS with a central opening, approximately $0.6 \mathrm{~cm}$ in diameter. Consequently, treatment with $100 \mathrm{~J}$ of electrical synchronised cardioversion was initiated, the patient then developed ventricular fibrillation, was defibrillated with $200 \mathrm{~J}$ and returned to sinus rhythm. Due to clinical progress and asymptomatic condition, the patient was discharged with a proposal to continue anticoagulant and antiarrhythmic medications and schedule an outpatient follow-up with a cardiologist. 
Figure 1: Electrocardiogram showing atypical atrial flutter with variable atrioventricular block

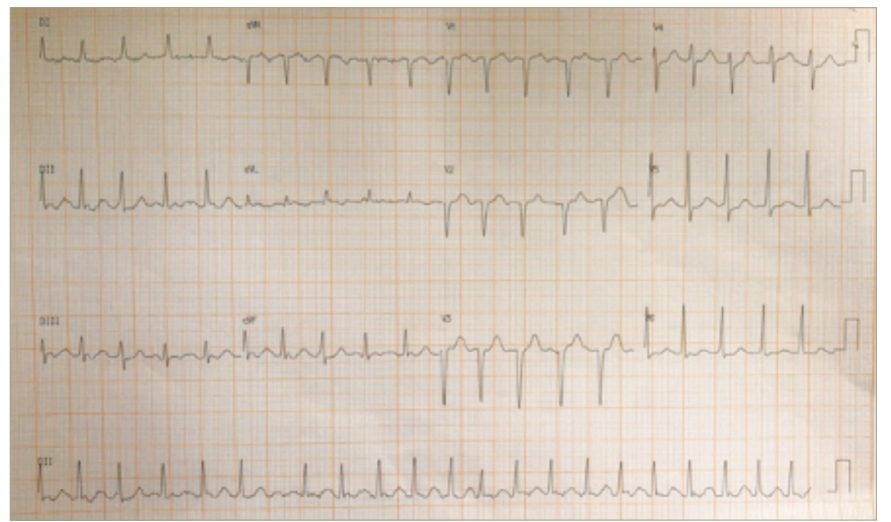

Figure 2: Posteroanterior chest X-ray - normal chest radiograph

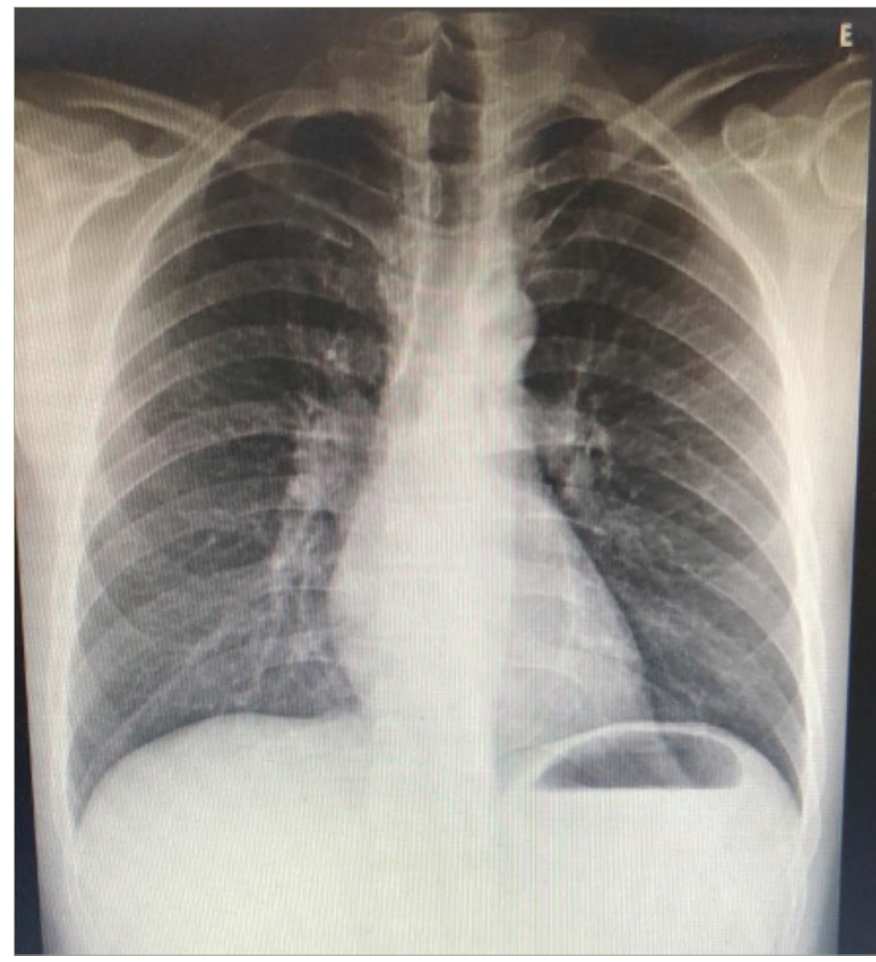

\section{Discussion}

CTS can occur in isolation or, even more frequently, with other congenital heart diseases. ${ }^{12}$ The referred patient, however, did not present other associated innate alterations. Several classifications have been proposed throughout history, among which, the most utilised was formulated by Loeffler, who divided the CTS into three distinct groups:

1. patients without openings in their membranes;

2. patients with one or more small openings in their membranes; and

3. patients with wide openings in their membranes. ${ }^{13}$

The non-manifestation of the disease in childhood is due to the remaining large communication between the left atrial chambers that allows the necessary haemodynamic circulatory exchange. ${ }^{4}$ Overall, those in the third group are likely to survive to late adulthood and often show little or no evidence of the disease. ${ }^{9,14}$ With regards to the patient concerned in this case report, he is classified as Loeffler grade III due to the wide opening
Figure 3: Transthoracic echocardiogram showing left intra-atrial membrane

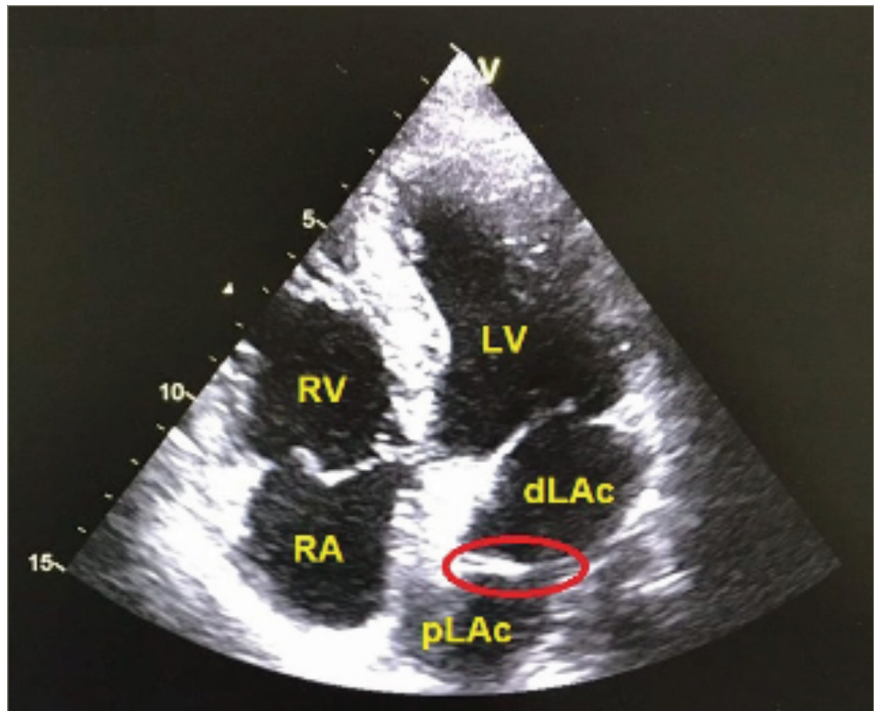

Red circle: left intra-atrial membrane.

$d L A C=$ distal left atrium chamber; $L V=$ left ventricle; $P L A C=$ proximal left atrium chamber; $R A$ = right atrium; $R V=$ right ventricle.

Figure 4: Transthoracic doppler echocardiography showing flow through atrial membranes

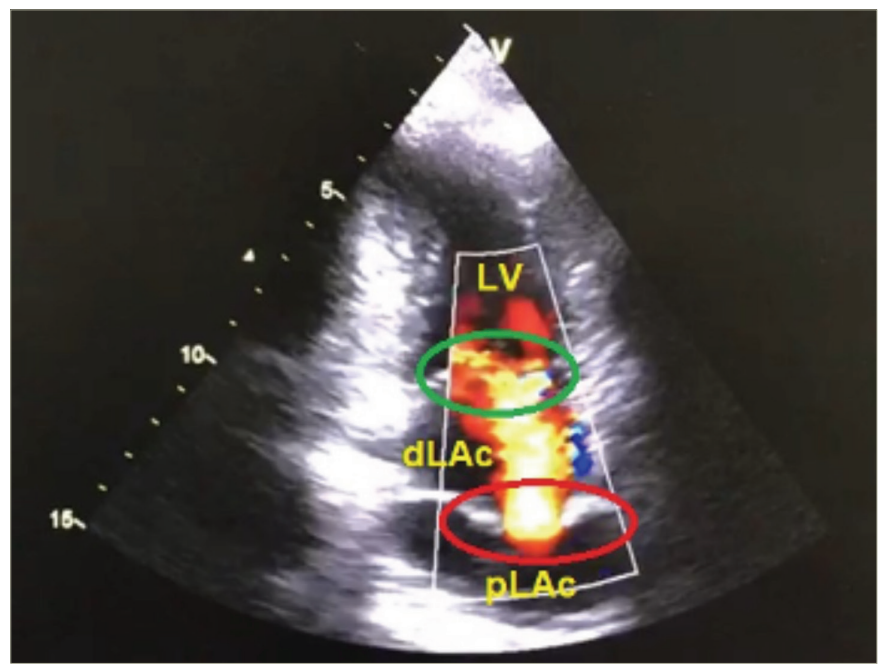

Red circle: left intra-atrial membrane. Green circle: mitral valve.

$d L A C=$ distal left atrium chamber; $L V=$ left ventricle; $p L A C=$ proximal left atrium chamber.

in his membrane associated with minimal peak gradient between the intra-atrial chambers and its manifestation in adulthood.

Clinical presentation in previously asymptomatic adults with CTS may occur and be caused by fibrosis and calcification at the membrane opening, leading to narrowing, obstruction and mimicking of mitral stenosis. In addition, the increased incidence of mitral regurgitation with advancing age may also contribute to the development of atrial fibrillation. ${ }^{15}$ Atrial arrythmias are common in CTS, with atrial fibrillation being described in $30 \%$ of studies on adults with CTS. ${ }^{8}$ Nevertheless, only two cases were found in the literature concerning CTS with atrial flutter. ${ }^{16,17}$ It is known that atrial flutter can be caused by several structural conditions, including mitral stenosis and regurgitation, which can be mimicked in CTS. ${ }^{16}$ The patient in this case developed signs and symptoms in adulthood, including atrial flutter and variable 
atrioventricular block, as well as signs of mitral valve regurgitation; therefore, there are slight similarities with the pathophysiology described in the literature.

The diagnosis of CTS can be established through imaging examinations such as transthoracic echocardiogram and transoesophageal echocardiography, which may be necessary to characterise, delineate and differentiate it from other cardiac anomalies, for example, atrial septal defect and supravalvular mitral annulus. ${ }^{18,19}$ The natural history of CTS is clinical stability without progressive left atrial obstruction, especially in patients with CTS without other congenital alterations and in those with an initial diagnosis made in adulthood. ${ }^{20}$ Thus, in older adults, comorbidity control and clinical follow-up alone may be sufficient. ${ }^{15}$ Surgery is the definitive treatment for this congenital condition, and, for adults, it has been indicated for symptomatic patients despite pharmacological treatment. The traditional technique consists of total resection of the left intra-atrial fibrous membrane and remains the gold standard in the management of symptomatic CTS. ${ }^{21,22}$

Due to the classic features of the disease, the rarity of this case is even more surprising, owing to the range of unique signs and symptoms. The proposed initial management and persistence of symptoms led to the need for more complex examinations, which were crucial for the establishment of accurate diagnosis and specific therapy. Outpatient follow-up with a cardiologist is a fundamental part of this patient's treatment, since the underlying cause of the disease was not altered, but controlled. Therefore, the patient is to be routinely monitored, so that if any changes in the patient's condition are spotted in the future, the choice of therapy for the patient can be re-evaluated accordingly. $\square$
1. Church WS. Congenital malformation of heart: abnormal septum in the left auricle. In: Transactions of the Pathological Society of London. 1868;19:188-90.

2. Hamdan R, Mirochnik N, Celermajer D, et al. Cor triatriatum sinister diagnosed in adult life with three dimensional transesophageal echocardiography. BMC Cardiovasc Disord. transesopha:

3. Ferreira SMA, Ferreira AG, Meguins LC, et al. Asthma as a clinical presentation of cor triatriatum sinister in a Brazilian amazon child: a case report. J Cardiovasc Med. 2009;10:795-7.

4. Fowler JK. Membranous band in the left auricle. In: Transactions of the Pathological Society of London. 1881;33:77-94.

5. Parsons CG. Cor triatriatum; concerning the nature of an anomalous septum in the left auricle. Br Heart J. 1950;10:327-38

6. Van praagh R, Corsini I. Cor triatriatum: pathologic anatomy and a consideration of morphogenesis based on 13 postmortem cases and a study of normal development of the pulmonary vein and atrial septum in 83 human embryos. Am Heart J. vein and atrial septir

7. Gharagozloo F, Bulkley BH, Hutchins GM. A proposed pathogenesis of cor triatriatum: impingement of the left superior vena cava on the developing left atrium.
Am Heart J. 1977;94:618-26

Zepeda IA, Morcos $P$, Castellanos LR. Cor triatriatum sinister identified after new onset atrial fibrillation on an elderly man. Case Rep Med 2014:2014:674018.

9. Krasemann Z, Scheld HH, Tjan D, et al. Cor triatriatum: short review of the literature upon ten new cases. Herz Kardiovask Erkrank. 2007:32:506-10.

10. D'Aloia A, Vizzardi E, Caretta G, et al. Diagnosis of cor triatriatum sinister in patient with pulmonary edema and severe pulmonary arterial hypertension: assessment by three-dimensional transesophageal echocardiography. Echocardiogr. 2011;28:198-201.

11. Narayanapillai J. Cor triatriatum sinister with severe obstruction: a rare presentation in an adult. BMJ Case Rep. 2016;2016:bcr2016215718.

12. Nassar $\mathrm{N}$, Hamdan RH. Cor triatriatum sinistrum: classification and imaging modalities. Eur I Cardiovasc Med 2011:1:84-7.

13. Loeffler E. Unusual malformations of the left atrium. Arch Pathol Lab Med. 1949;48:371-6.

14. Chen $\mathrm{Q}$ Guhathakurta S, Vadalapali G, et al. Cor triatriatum in adults: three new cases and a brief review. Tex Heart Inst $J$. 1999:26:206-10.

15. Slight $D$, Nzewi $O C$, Buell $R$, et al. Cor-triatriatum sinister presenting in the adult as mitral stenosis: an analysis of factors which may be relevant in late presentation. Heart Lung Circ. 2005;14:8-12.

16. Borne RT, Gonzalez J, Khanna A, et al. Getting to the right left atrium: catheter ablation of atrial fibrillation and mitral annular flutter in cor triatriatum. HeartRhythm Case Rep. 2016;2:502-5.

17. Maged FN, Colin TW, Eric TC. Ablation of isthmus and non-isthmus-dependent flutters in a patient with cor triatriatum dexter. Europace. 2016;15:1573.

18. Malik A, Fram D, Mohani $A$, et al. Cor triatriatum: a multimodality imaging approach. Can J Cardiol. 2008;24:19-20.

19. Jha AK, Makhja N. Cor triatriatum: a review. Semin Cardiothorac Vasc Anesth. 2017;21:178-85.

20. Modi KA, Annamali S, Ernest K, et al. Diagnosis and surgical correction of cor triatriatum in an adult: combined use of transesophageal and contrast echocardiography, and a review of literature. Echocardiogr. 2006;23:506-9.

21. Fuchs MM, Connolly HM, Said SM, et al. Outcomes in patients with cor triatriatum sinister. Congenit Heart Dis. 2018:13:628-32.

22. Humpl T, Reineker K, Manlhiot C, et al. Cor triatriatum sinistrum in childhood. A single institution's experience. Can I Cardiol. 2010;26:371-6. 